\title{
Retrieving the three-dimensional matter power spectrum and galaxy biasing parameters from lensing tomography (Corrigendum)
}

\author{
Patrick Simon \begin{abstract}
e-mail: psimon@astro.uni-bonn.de
\end{abstract} \\ Argelander-Institut für Astronomie, Universität Bonn, Auf dem Hügel 71, 53121 Bonn, Germany
}

A\&A 543, A2 (2012), https://doi.org/10.1051/0004-6361/201118224

Key words. methods: data analysis - large-scale structure of Universe - dark matter - errata, addenda

We report a missing factor, $a(\chi)$, in the $\xi_{ \pm}$-related integrands of the original paper. This typographical error in Eqs. (11), (16), and (17) has no impact on our results and conclusions; the correct integrands were used in the original analysis.

A detailed list of the three typographical errors follows. First, the integral for the shear-shear correlations in Eq. (11) should have the factor $a^{2}(\chi)$ in the denominator of the integrand (instead of just $a(\chi)$ ),

$\xi_{ \pm}^{(i j)}(\theta)=$

$$
\frac{9 H_{0}^{4} \Omega_{\mathrm{m}}^{2}}{4 c^{4}} \int_{0}^{\chi_{\mathrm{h}}} \int_{0}^{\infty} \frac{\mathrm{d} \chi \mathrm{d} \ell \ell}{2 \pi} \frac{\bar{W}^{(i)}(\chi) \bar{W}^{(j)}(\chi)}{a^{2}(\chi)} J_{0,4}(\ell \theta) P_{\delta}\left(\frac{\ell}{f_{\mathrm{k}}(\chi)}, \chi\right) .
$$

Second, the integral for the basis functions in Eq. (16) should also have the factor $a^{2}(\chi)$,

$$
\begin{aligned}
& X_{ \pm}^{(i j)}(\theta ; m, n):=\frac{9 H_{0}^{4} \Omega_{\mathrm{m}}^{2}}{8 \pi c^{4} \theta^{2}} \times \\
& \int_{\chi_{n}}^{\chi_{n+1}} \mathrm{~d} \chi \frac{\bar{W}^{(i)}(\chi) \bar{W}^{(j)}(\chi)}{a^{2}(\chi)} \int_{k_{m} f_{\mathrm{k}}(\chi) \theta}^{k_{m+1} f_{\mathrm{k}}(\chi) \theta} \mathrm{d} s s J_{0,4}(s) P_{\delta}^{\mathrm{fid}}\left(\frac{s}{f_{\mathrm{k}}(\chi) \theta}, \chi\right) .
\end{aligned}
$$

Third, the integral in Eq. (17) must have the factor $a^{2}(\chi)$,

$$
\begin{aligned}
& \xi_{ \pm, \mathrm{fid}}^{(i j)}(\theta):=\frac{9 H_{0}^{4} \Omega_{\mathrm{m}}^{2}}{8 \pi c^{4} \theta^{2}} \times \\
& \int_{0}^{\chi \mathrm{h}} \mathrm{d} \chi \frac{\bar{W}^{(i)}(\chi) \bar{W}^{(j)}(\chi)}{a^{2}(\chi)} \int_{0}^{\infty} \mathrm{d} s s J_{0,4}(s) P_{\delta}^{\mathrm{fid}}\left(\frac{s}{f_{\mathrm{k}}(\chi) \theta}, \chi\right) .
\end{aligned}
$$

All three of the correct equations shown here were used in our original analysis. Therefore, the corrections have no impact on our results and conclusions of the original paper. 